\title{
On the Born-Oppenheimer Approximation of Wave Operators in Molecular Scattering Theory
}

\author{
M. Klein ${ }^{1, \star}$, A. Martinez ${ }^{2}$, and X.P. Wang ${ }^{3, \star \star}$ \\ 1 Department of Physics, Technion, 3200 Haifa, Israel \\ 2 Département de Mathématiques et d'Informatique, Université de Paris-Nord, \\ Avenue Jean-Baptiste Clément, F-93430 Villetaneuse, France \\ 3 Département de Mathématiques, Université de Nantes, F-44072 Nantes Cedex 03, France
}

Received January 7, 1992; in revised form May 27, 1992

\begin{abstract}
In this paper we study the diatomic molecular scattering by reducing the number of particles through Born-Oppenheimer approximation. Under a nontrapping assumption on the effective potential of the molecular Hamiltonian we use semiclassical resolvent estimates to show that non-adiabatic corrections to the adiabatic (or Born-Oppenheimer) wave operators are small. Furthermore we study the classical limit of the adiabatic wave operators by computing its action on quantum observables microlocalized by use of coherent states.
\end{abstract}

\section{Introduction}

In this work, we are concerned with a mathematical study of the scattering process that a diatomic molecule dissociates into two ions. A direct mathematical study of this problem may be rather difficult, since the number of particles can be arbitrarily large. However, there is a folk-theorem in molecular scattering theory (see e.g. [Ch, De, WO]) that the contribution of electrons can be taken care of by effective potentials and the motion of nuclei can be well approximated by classical dynamics. This indicates that one can reduce the number of particles and thus considerably simplify the problem. This beautiful physical intuition dates back to Born-Oppenheimer [BO] and is based on the existence of a natural small parameter in molecular Schrödinger operators: the ratio of the mass of the electron to the mass of the nucleus. It is surprising to us that until now, there is no rigorous mathematical work on BornOppenheimer approximation in scattering theory, unless the attempt made in [Ra] where no quantitative result was given. Concerning mathematical works on BornOppenheimer approximation in spectral theory, the reader can track back from the reference quoted in [KMSW].

\footnotetext{
* Permanent address: Fachbereich Mathematik MA 7-2, Technische Universität Berlin, 1000 Berlin 12, FRG

$\star \star$ On leave from: Departement of Mathematics, Peking University, 100871 Beijing, China
} 
The purpose of this paper is to provide a mathematical justification of BornOppenheimer approximation in scattering theory for a simple, particular scattering quantity: the two-cluster wave operators in diatomic molecular scattering. To begin with, let us introduce some notations. Consider the Schrödinger operator $P$ obtained after the removal of mass center from the energy operator

$$
\widetilde{P}=-\frac{1}{2 m_{1}} \Delta_{x_{1}}-\frac{1}{2 m_{2}} \Delta_{x_{2}}+\sum_{j=3}^{N+2}-\frac{1}{2 m_{2}} \Delta_{x_{\jmath}}+\sum_{\imath<\jmath} V_{\imath \jmath}\left(x_{i}-x_{j}\right)
$$

of a diatomic molecule with $N$ electrons. Here $x_{1}, x_{2}$ (resp., $m_{1}, m_{2}$ ) denote the position (resp., the mass) of the two nuclei in $\mathbf{R}^{n}$ and $x_{\jmath}, j \geq 3$, (resp., $m$ ) denote the position (resp., the mass) of electrons, the Planck's constant $\hbar$ being put to 1 . In the following, we assume that $m_{1}, m_{2}$ are of the same order and are all large enough compared with $m$. In this paper the interaction potentials $V_{\imath j}(x)$ are assumed to be short-range (a precise definition will be given below). Let $C=\left(C_{1}, C_{2}\right)$ be a two cluster decomposition of $\{1,2, \ldots, N+2\}$ with $j \in C_{j}, j=1,2 .\left|C_{j}\right|$ will denote the number of particles in the cluster $C_{j}$. Then the total Hamiltonian $P$ can be decomposed as

$$
P=P_{0}+P^{c}+I_{c},
$$

where $P_{0}$ is the kinetic energy of the relative motion of the centers of mass of the sub-systems $C_{1}$ and $C_{2}$ :

$$
P_{0}=-\left(\frac{1}{2 M_{1}}+\frac{1}{2 M_{2}}\right) \Delta_{x}, \quad x \in \mathbf{R}^{n}
$$

where $M_{j}=m_{j}+\left(\left|C_{j}\right|-1\right) m, j=1,2$, is the total mass of cluster $C_{j}, P^{c}$ is the cluster Hamiltonian whose detailed expression depends on the choice of cluster coordinates associated to $C=\left(C_{1}, C_{2}\right)$ and $I_{c}$ is the intercluster interaction:

$$
I_{c}=\sum_{\imath \in C_{1}, j \in C_{2}} V_{i j}\left(x_{\imath}-x_{j}\right)
$$

expressed in the coordinates $(x, y), x \in \mathbf{R}^{n}, y \in \mathbf{R}^{N n}$, where $y$ are the intra-cluster coordinates. Since $m_{1}, m_{2} \gg m$, it is natural to regard the inverse of reduced masses as a small parameter. We henceforth set $m=1$ and put

$$
\left(\frac{1}{2 M_{1}}+\frac{1}{2 M_{2}}\right)=h^{2}, \quad h>0,
$$

and

$$
P_{c}=P_{0}+P^{c}=-h^{2} \Delta_{x}+P^{c} .
$$

In this paper, we regard $h>0$ as a small parameter and we want to study the BornOppenheimer approximation (i.e. the limit $h \rightarrow 0$ ) of the two-cluster wave operators

$$
\Omega_{ \pm}^{c}=\mathrm{s}^{-} \lim _{t \rightarrow \pm \infty} e^{i t P} e^{-i t P_{c}} E_{p}\left(P^{c}\right)
$$

in $L^{2}\left(\mathbf{R}^{n(N+1)}\right)$, where $E_{p}\left(P^{c}\right)$ is the spectral projection in $L^{2}\left(\mathbf{R}^{n(N+1)}\right)$ associated to the point spectrum of $P^{c}$. Analogous to the Born-Oppenheimer approximation in spectral theory (see [CSD, KMSW]), we denote by $P_{e}$ the electronic Hamiltonian

$$
P_{e}=P^{c}+I_{c}(x, y)
$$


which can be decomposed as a direct integral in $x$-variables. But, in contrast to the Born-Oppenheimer approximation in spectral theory, $x$ now is not the relative position between the nuclei, but that between the two centers of mass of the subsystems $C_{1}$ and $C_{2}$. In addition, because of the choice of cluster coordinates, the intercluster interaction $I_{c}$ is now $h$-dependent. Thus we have to analyze the $h$-dependence of the eigenvalues of $P_{e}(x)=P^{c}+I_{c}(x, \cdot)$.

Let us now explain our approach to the Born-Oppenheimer approximation of $\Omega_{ \pm}^{c}$. Fix an eigenvalue $E_{0}$ of the cluster Hamiltonian $P^{c}$. Assume that $E_{0}$ is in discrete spectrum of $P^{c}$ with multiplicity $m$ and that there exist exactly $m$ curves of eigenvalues of $P_{e}(x), \lambda_{1}(x), \ldots, \lambda_{m}(x)$, counted according to their multiplicity, which converge to $E_{0}$ as $x \rightarrow \infty$. Let $\Pi_{0}$ and $\Pi(x)$ denote the spectral projections of $P^{c}$ and $P_{e}(x)$ associated to $E_{0}$ and $\lambda_{1}(x), \ldots, \lambda_{m}(x)$ respectively. Denote by $\Pi_{0}$ and $\Pi$ the projections in the total space $L^{2}\left(\mathbf{R}^{n(N+1)}\right)$ induced by the action of $\Pi_{0}$ and $\Pi(x), x \in \mathbf{R}^{n}$, in the space $L^{2}\left(\mathbf{R}^{n N}\right)$ of the intra-cluster coordinates. Note that $\Pi_{0}$ (acting on $L^{2}\left(\mathbf{R}^{n(N+1)}\right)$ ) is always of infinite rank, since it commutes with translations in $x$. Define the adiabatic and non-adiabatic parts of $P$ by

$$
P^{A D}=\Pi P \Pi, \quad Q^{A D}=\widehat{\Pi} P \widehat{\Pi}, \quad \widehat{\Pi}=1-\Pi .
$$

Under appropriate assumptions on the potentials, we can show that on the range of $\Pi_{0}$, the cluster wave operators $\Omega_{ \pm}^{c}$ have a decomposition:

$$
\Omega_{ \pm}^{c} \Pi_{0}=\Omega_{ \pm}^{N A D} \Omega_{ \pm}^{A D}
$$

where

$$
\Omega_{ \pm}^{A D}=\mathrm{s}-\lim _{t \rightarrow \pm \infty} e^{\imath t P^{A D}} e^{-i t P_{c}} \Pi_{0}
$$

and

$$
\Omega_{ \pm}^{N A D}=\mathrm{s}-\lim _{t \rightarrow \pm \infty} e^{i t P} e^{-i t P^{A D}} E_{\mathrm{ac}}\left(P^{A D}\right)
$$

where $E_{\mathrm{ac}}\left(P^{A D}\right)$ is the spectral projection onto the absolutely continuous spectral subspace $\mathscr{H}_{a \mathrm{c}}\left(P^{A D}\right)$ of $P^{A D}$. It can be shown under rather general conditions that $\Omega_{ \pm}^{A D}$ and $\Omega_{ \pm}^{N A D}$ exist and are complete (see Theorem 2.3 and also [Ra]). With these preparations, the main results of this paper can be summarized as follows:

- Under a non-trapping assumption on $P^{A D}$, one has:

$$
\Omega_{ \pm}^{N A D}-1=O(h)
$$

in the norm of bounded operators (Theorem 4.1).

- If $\Pi$ is of rank one, $\Omega_{ \pm}^{A D}$ "converges" in the limit $h \rightarrow 0$ to the classical wave operators of the classical effective Hamiltonians (Theorem 5.3, 5.4).

The first part of the results shows that $\Omega_{ \pm}^{A D}$ gives a good approximation of $\Omega_{ \pm}^{c} \Pi_{0}$, while the second part justifies the physical intuition that the motion of heavy particles can be well approximated by classical mechanics. Combining these two parts, we obtain a quantitative result of the cluster wave operators $\Omega_{ \pm}^{c}$ in the limits $h \rightarrow 0$ (see Theorem 5.5).

This work is organized as follows. In Sect. 2, we formulate some results on adiabatic and non-adiabatic wave operators. These results are stimulated by the unpublished work [Ra]. In Sect. 3, we establish semiclassical resolvent estimates for $P^{A D}(h)$ and $P(h)$. We need a non-trapping assumption on the classical effective Hamiltonian. This condition is satisfied according to $[\mathrm{DH}]$ in a slightly different situation. In Sect. 4, we derive the adiabatic approximation of the cluster wave 
operators. Our result is strong, since we obtain a remainder estimate in the operator norm. But we need the non-trapping condition to control the $h$-dependence of quantum dynamics. In Sect. 5, we study the classical limit of the adiabatic wave operators. The method is similar to [W3]: We construct incoming and outgoing parametrices with operator-valued symbols to approximate the adiabatic wave operators. We remark that we use the phase function constructed in [W3], which is different from that of [IK] and has the advantage of being directly related to the classical wave operators. In the main part of Sect. 5, we do not need the non-trapping assumption, except for the last result Theorem 5.5 .

The methods used in this paper vary with sections. Those in Sects. 2-4 are relatively elementary: We mainly use methods from functional analysis, except for Corollary 3.3, where the symbolic calculus of h-pseudo-differential operators enters. Therefore we expect that the main results of these sections could be understood by those having a knowledge of quantum two-body short-range scattering theory (including Mourre's estimates and Kato's local smoothness theory). However, we require in Sect. 5 a more developed mathematical tool: theory of outgoing and incoming h-parametrices. The proofs in Sect. 5 rely heavily on the results of earlier works [IK] (for $h=1$ ), [W1] and, especially, [W3]. We suggest that the reader who is interested in the details of this section read these works.

\section{Some Preliminaries}

In this section, we establish the decomposition for the cluster wave operators:

$$
\Omega_{ \pm}^{c} \Pi_{0}=\Omega_{ \pm}^{N A D} \Omega_{ \pm}^{A D} .
$$

The similar problem was studied in [Ra] for Coulomb potentials with specific nuclear charges: One of the ions is assumed neutral. Our framework is slightly different: We treat general, short-range potentials.

To study the Born-Oppenheimer approximation of the cluster wave operators, it is necessary to investigate the relationship between the electronic Hamiltonian $P_{e}(x)$ and the cluster Hamiltonian $P^{c}$. We shall write down the expressions of $P_{e}(x)$ and $P^{c}$ in clustered atomic coordinates. One could equally well use clustered Jacobi coordinates instead. But then the parameter-dependence in the potentials becomes more complicated (see [Ra]). Let $C=\left(C_{1}, C_{2}\right)$ be a cluster decomposition with $j \in C_{j}, j=1,2$. Let $C_{j}^{\prime}=C_{j} \backslash\{j\}$. In the center-of-mass subspace

$$
R \equiv \frac{1}{m_{1}+m_{2}+N}\left(m_{1} x_{1}+m_{2} x_{2}+\sum_{\jmath=3}^{N+2} x_{j}\right)=0
$$

of $\mathbf{R}^{n N} \times \mathbf{R}^{n} \times \mathbf{R}^{n}$, we introduce new coordinates $(y, x) \in \mathbf{R}^{n N} \times \mathbf{R}^{n}$ by setting

$$
\begin{aligned}
y_{\jmath} & =x_{j}-x_{k}, \quad \text { for } j \in C_{k}^{\prime}, \quad k=1,2, \\
x & =R_{1}-R_{2}, \\
R_{\jmath} & =\frac{1}{M_{j}}\left(m_{j} x_{\jmath}+\sum_{k \in C_{\jmath}^{\prime}} x_{k}\right), \quad M_{\jmath}=m_{j}+|C|_{j}^{\prime} \mid, \quad j=1,2 .
\end{aligned}
$$

Here the mass of electrons is set to 1 . Recall that $x_{1}$ and $x_{2}$ stand for the position of the two nuclei. Thus, in the $(y, x)$ coordinates, we measure the position of the 
electrons inside each ion (= cluster) by their relative position with respect to the nucleus. With this choice of coordinates, we have

$$
\begin{aligned}
P= & -h^{2} \Delta_{x}+P_{e}(x), \quad h^{2}=\frac{1}{2 M_{1}}+\frac{1}{2 M_{2}}, \\
P_{e}(x)= & P^{c}+I_{c}(x), \\
P^{c}= & \sum_{k=1}^{2}\left\{\sum_{j \in C_{k}^{\prime}}\left(-\Delta_{y_{j}}+V_{k j}\left(y_{j}\right)\right)+\frac{1}{m_{k}}\left(\sum_{j \in C_{k}^{\prime}} D_{y_{j}}\right)^{2}+\sum_{i, j \in C_{k}^{\prime}} V_{\imath j}\left(y_{i}-y_{j}\right)\right\}, \\
I_{c}(x)= & \sum_{i \in C_{1}^{\prime}, j \in C_{2}^{\prime}} V_{\imath j}\left(y_{i}-y_{j}+x-f_{1}+f_{2}\right)+\sum_{j \in C_{1}^{\prime}} V_{2 j}\left(-x+f_{1}-f_{2}-y_{j}\right) \\
& +\sum_{j \in C_{2}^{\prime}} V_{1 j}\left(x-f_{1}+f_{2}-y_{j}\right)+V_{12}\left(x-f_{1}+f_{2}\right) .
\end{aligned}
$$

Here

$$
f_{j}=\frac{1}{M_{\jmath}} \sum_{k \in C_{\jmath}^{\prime}} y_{k} .
$$

Note that $f_{j}$ is of the order $O\left(h^{2}|y|\right)$.

Using the notation $\langle x\rangle=\left(1+x^{2}\right)^{1 / 2}$, our general assumptions on the potentials $V_{i j}, 1 \leq i, j \leq N+2$, are the following:

$V_{i j}$ is smooth on $\mathbf{R}^{n} \backslash\{0\}$ and is of short-range:

$$
\begin{gathered}
\left|\partial_{x}^{\alpha} V_{i j}(x)\right| \leq C_{\alpha}\langle x\rangle^{-\varrho-|\alpha|}, \quad \forall \alpha \in \mathbf{N}^{n}, \quad|x|>1, \quad \text { for some } \varrho>1, \\
V_{\imath j} \text { is } \Delta \text {-compact. }
\end{gathered}
$$

This in particular covers the case of Coulomb singularities $V_{i j}(x) \sim|x|^{-1}$ for $x$ near 0 in $\mathbf{R}^{n}, n \geq 3$. Conditions (2.7) and (2.8) will be replaced by a stronger condition (3.1) later on. As a first result, we state the following

Lemma 2.1. Under the assumptions (2.7) and (2.8) one has

$$
\lim _{|x| \rightarrow \infty} P_{e}(x)=P^{c}
$$

in the sense of strong resolvent convergence in $L^{2}\left(\mathbf{R}^{n N}\right)$.

Proof. It suffices to remark that s- $\lim _{|x| \rightarrow \infty} I_{c}(x)\left(P^{c}+i\right)^{-1}=0$ in $L^{2}\left(\mathbf{R}^{n N}\right)$.

Let $\Sigma(x)=\inf \sigma_{\text {ess }}\left(P_{e}(x)\right)$. Put $\Sigma_{0}=\liminf \Sigma(x)$. Let $E_{0} \in \sigma_{d}\left(P^{c}\right)$ be an eigenvalue of $P^{c}$ with $E_{0}<\Sigma_{0}$. Assume that $E_{0}$ is of multiplicity $m$. By Lemma 2.1, every point in the spectrum $\sigma\left(P^{c}\right)$ is a limit of elements in $\sigma\left(P_{e}(x)\right.$ ) (see [K]). In addition, by the exceptional decay of eigenfunctions of $P^{c}$ associated with eigenvalues $E_{0}$ (see [A]), we can show that there are at least $m$ curves of eigenvalues of $P_{e}(x)$, counted with their multiplicity, which tend to $E_{0}$ as $x \rightarrow \infty$. In this paper, we make the following stability assumption on $E_{0}$. We shall say that $E_{0}$ is stable, if

there are exactly $m$ curves of eigenvalues of $P_{e}(x)$ which converge to $E_{0}$.

Note that this assumption is satisfied by a $\mathrm{H}_{2}^{+}$-like molecule with generic unequal nuclear charges. See Appendix A in [Ra]. 
Under the assumption (2.9), we can find an $\varepsilon>0$ sufficiently small such that

$$
\sigma\left(P_{e}(x)\right) \cap\left\{z \in \mathbf{C} ; \varepsilon / 2 \leq\left|z-E_{0}\right| \leq 2 \varepsilon\right\}=\emptyset, \quad \text { for } \quad|x|>R_{0},
$$

with $R_{0}$ sufficiently large. Consequently we can define the spectral projections

$$
\begin{aligned}
\Pi_{0} & =\frac{1}{2 \pi i} \oint_{\left|z-E_{0}\right|=\varepsilon}\left(z-P^{c}\right)^{-1} d z, \\
\Pi(x) & =\frac{1}{2 \pi i} \oint_{\left|z-E_{0}\right|=\varepsilon}\left(z-P_{e}(x)\right)^{-1} d z
\end{aligned}
$$

for $|x|>R_{0}$. Lemma 2.1 gives that s- $\lim _{|x| \rightarrow \infty} \Pi(x)=\Pi_{0}$. We have the following much stronger result.

Theorem 2.2. Under the assumptions (2.7), (2.8) and (2.9), one has

$$
\left\|\partial_{x}^{\alpha}\left(\Pi(x)-\Pi_{0}\right)\right\|=O\left(\langle x\rangle^{-|\alpha|-\varrho}\right)
$$

for $|x|>R_{0}, \alpha \in \mathbf{N}^{n}$ with $|\alpha| \leq 2$. Here $\varrho>1$ is the same as in (2.7).

Proof. We begin with proving that

$$
\left\|\partial_{x}^{\alpha}\left(\Pi(x)-\Pi_{0}\right) \Pi_{0}\right\|=O\left(\langle x\rangle^{-|\alpha|-\varrho}\right)
$$

for $|x|>R_{0}, \alpha \in \mathbf{N}^{n}$ with $|\alpha| \leq 2$. To do this, we use the identity

$$
\Pi(x)-\Pi_{0}=\frac{1}{2 \pi i} \oint_{\left|z-E_{0}\right|=\varepsilon}\left(z-P_{e}(x)\right)^{-1} I_{c}(x)\left(z-P^{c}\right)^{-1} d z .
$$

Since $\left\|\left(z-P_{e}(x)\right)^{-1}\right\| \leq 2 / \varepsilon$ uniformly in $x$ and by the exponential decay of the eigenfunctions of $P^{c},\left\|I_{c}(x) \Pi_{0}\right\| \leq C\langle x\rangle^{-\varrho}$, we obtain (2.13) for $\alpha=0$. The result for $\alpha \in \mathbf{N}^{n}$ with $|\alpha| \leq 2$ follows from the arguments used above and the conditions (2.7) and (2.8). Note that the argument of [CDS] shows that the map $x \mapsto \Pi(x)$ is twice continuously differentiable for $|x|>R_{0}$.

To prove (2.12), we use the equalities:

$$
\begin{aligned}
\Pi(x)-\Pi_{0} & =\left(\Pi(x)-\Pi_{0}\right)^{2}-2 \Pi_{0}+\Pi(x) \Pi_{0}+\Pi_{0} \Pi(x) \\
& =\left(\Pi(x)-\Pi_{0}\right)^{2}+\left(\Pi(x)-\Pi_{0}\right) \Pi_{0}+\Pi_{0}\left(\Pi(x)-\Pi_{0}\right) .
\end{aligned}
$$

According to the stability assumption, we have: $\lim _{|x| \rightarrow \infty}\left\|\left(\Pi(x)-\Pi_{0}\right)\right\|=0$. See $[\mathrm{K}]$. So $\left\|\left(\Pi(x)-\Pi_{0}\right)^{2}\right\| \leq 1 / 2\left\|\Pi(x)-\Pi_{0}\right\|$ for $|x|$ large enough. Since $\left\|\left(\Pi(x)-\Pi_{0}\right) \Pi_{0}\right\|=$ $\left\|\Pi_{0}\left(\Pi(x)-\Pi_{0}\right)\right\|$, we obtain from (2.15) that

$$
\left\|\Pi(x)-\Pi_{0}\right\| \leq 4\left\|\left(\Pi(x)-\Pi_{0}\right) \Pi_{0}\right\|,
$$

for $|x|$ large enough. Equation (2.12) for $\alpha=0$ follows from (2.13). Equation (2.12) for $|\alpha| \leq 2$ can be derived from (2.13) by differentiating (2.15).

Let $\lambda_{1}(x), \ldots, \lambda_{m}(x)$ denote these eigenvalues of $P_{e}(x)$ that converge to $E_{0}$ as $|x| \rightarrow \infty$. From Theorem 2.2, we derive that:

$$
\lambda_{j}(x)-E_{0}=O\left(\langle x\rangle^{-\varrho}\right), \quad j=1,2, \ldots, m,
$$


for $|x|$ large enough. We assume that the map $x \mapsto \lambda_{j}(x) \in \sigma_{d}\left(P_{e}(x)\right)$ is globally defined on $\mathbf{R}^{n}$ for $j=1, \ldots, m$ (in particular, there is no absorption into the essential spectrum of $\left.P_{e}(x)\right)$ and

$$
\operatorname{dist}\left(\sigma\left(P_{e}(x)\right) \backslash\left\{\lambda_{1}(x), \ldots, \lambda_{m}(x)\right\},\left\{\lambda_{1}(x), \ldots, \lambda_{m}(x)\right\}\right) \geq \delta>0
$$

for all $x \in \mathbf{R}^{n}$. It then follows from the argument in [CDS] that the associated projection $\Pi(x)$ is of class $C^{2}$ on $\mathbf{R}^{n}$. Note that we can always take $\lambda_{\jmath}(x)$ to be continuous and, if $m=1$, to be of class $C^{2}$.

Now let $P^{A D}, Q^{A D}$ and $\Omega_{ \pm}^{A D}, \Omega_{ \pm}^{N A D}$ be defined as in the Introduction. We shall say that the wave operators $\Omega_{ \pm}^{A D}$ (resp. $\Omega_{ \pm}^{N A D}$ ) are complete if the range of $\Omega_{ \pm}^{A D}$ is equal to the absolutely continuous spectral space of $P^{A D}: R\left(\Omega_{ \pm}^{A D}\right)=\mathscr{H}_{\text {ac }}\left(P^{A D}\right)$ (resp., if $R\left(\Omega_{ \pm}^{N A D} E_{\mathrm{ac}}\left(P^{A D}\right)\right)=R\left(\Omega_{ \pm}^{c} \Pi_{0}\right)$ ). In the following, we shall only consider the case $t \rightarrow+\infty$ and therefore omit the subscripts \pm .

Theorem 2.3. Under the assumptions of Theorem 2.2, assume in addition (2.16). Then the wave operator $\Omega^{A D}$ and $\Omega^{N A D}$ exist and are complete and one has:

$$
\Omega^{c} \Pi_{0}=\Omega^{N A D} \Omega^{A D} \text {. }
$$

Proof. The method is similar to usual two-body short-range scattering theory. Therefore we shall only sketch the argument. To study the existence and the completeness of $\Omega^{A D}$, we note that, using $\left[\Delta_{x}, \Pi_{0}\right]=0$ and Theorem 2.2,

$$
\begin{aligned}
\left(P^{A D}-P_{c}\right) \Pi_{0}= & -h^{2}\left(\left(\Pi(x)-\Pi_{0}\right) \Delta_{x} \Pi(x)+\Delta_{x} \Pi_{0}\left(\Pi(x)-\Pi_{0}\right)\right) \Pi_{0} \\
& +\left(\Pi(x) P_{e}(x) \Pi(x)-E_{0}\right) \Pi_{0}=O\left(\langle x\rangle^{-\varrho}\right)
\end{aligned}
$$

on the range of $\left(-\Delta_{x}+i\right)^{-1}$, with $\varrho>1$ given by (2.7). This shows that $P^{A D}$ is a short-range perturbation of $P_{c}$. The existence of $\Omega^{A D}$ then follows from Cook's method. To prove the completeness of $\Omega^{A D}$, we note that the continuous spectrum of $P^{A D}$ is given by: $\sigma_{c}\left(P^{A D}\right)=\left[E_{0}, \infty\right.$ [, and on this interval we can use Mourre's commutator method to establish the limiting absorption principle (see [Ra] and Sect. 3 of this paper). The asymptotic completeness of $\Omega^{A D}$ follows from stationary methods.

To study $\Omega^{N A D}$, we indicate that since the range $R\left(E_{\mathrm{ac}}\left(P^{A D}\right)\right) \subseteq R(\Pi)$, we have

$$
\begin{aligned}
\left(P-P^{A D}\right) E_{\mathrm{ac}}\left(P^{A D}\right) & =-h^{2} \widehat{\Pi} \Delta_{x} \Pi E_{\mathrm{ac}}\left(P^{A D}\right) \\
& =h^{2}\left[\Delta_{x}, \Pi\right] \Pi E_{\mathrm{ac}}\left(P^{A D}\right) .
\end{aligned}
$$

Theorem 2.2 shows that $\left[\Delta_{x}, \Pi(x)\right]$ is a first order differential operator with shortrange coefficients. Thus the existence of the wave operator again follows from Cook's method. To show the completeness of $\Omega^{N A D}$, we need to show that the limit

$$
\lim _{t \rightarrow \infty} e^{i t P^{A D}} e^{-\imath t P} \phi, \quad \forall \phi \in R\left(\Omega^{c} \Pi_{0}\right)
$$

exists and is in the absolutely continuous subspace $\mathscr{H}_{\mathrm{ac}}\left(P^{A D}\right)$ of $P^{A D}$. But since $\phi \in R\left(\Omega^{c} \Pi_{0}\right)$, one has that $\left\|e^{-i t P} \phi-e^{-i t P_{c}} \Pi_{0} \psi\right\| \stackrel{\text { ac }}{\rightarrow} 0$ for some $\psi$. Thus the completeness of $\Omega^{N A D}$ follows from the existence of the limit

$$
\lim _{t \rightarrow \infty} e^{i t P^{A D}} e^{-i t P} \phi=\lim _{t \rightarrow \infty} e^{i t P^{A D}} e^{-i t P_{c}} \Pi_{0} \psi
$$

which in turn follows from the existence of $\Omega^{A D}$. 
Theorem 2.3 says that we can split the two-cluster scattering in two processes: adiabatic and non-adiabatic scattering. To justify quantitatively the usefulness of the adiabatic (or Born-Oppenheimer) approximation in scattering theory, we shall study in the remaining sections the wave operators $\Omega^{A D}$ and $\Omega^{N A D}$ in the limit $h \rightarrow 0$.

\section{Resolvent Estimates}

As remarked in the Introduction, in the framework of two-cluster scattering the electronic Hamiltonian $P_{e}(x)$ is $h$-dependent: not only there is a perturbation of the kinetic part in $P_{e}(x)$ of order $O\left(h^{2}\right)$, but also the parameter $h$ appears in the interaction potentials, given by (2.6). To analyze the $h$-dependence of the eigenvalues of $P_{e}(x)$, we shall in this section impose the stronger hypothesis

$$
\begin{aligned}
& \text { All potentials } V_{\imath \jmath}, 1 \leq i, j \leq N+2 \text {, are smooth and short-range: } \\
& \left|\partial_{x}^{\alpha} V_{i j}(x)\right| \leq C_{\alpha}\langle x\rangle^{-\varrho-|\alpha|}, \quad \text { for some } \varrho>1, \forall x \in \mathbf{R}^{n} .
\end{aligned}
$$

The case of potentials with Coulomb-like singularities will be discussed elsewhere. We denote $P_{e}(x)$ and $P^{c}$ by $P_{e}(x, h)$ and $P^{c}(h)$, respectively, to single out the $h$ dependence of the Hamiltonians, and we refer to (2.4) for an explicit expression for $P_{e}(x, h)$ and $P^{c}(h)$. Put

$$
P_{e}(x, 0)=P^{c}(0)+I_{c}(x, 0),
$$

where

$$
P^{c}(0)=\sum_{k=1}^{2}\left\{\sum_{j \in C_{k}^{\prime}}\left(-\Delta_{y_{j}}+V_{k j}\left(y_{j}\right)\right)+\sum_{i, j \in C_{k}^{\prime}} V_{i j}\left(y_{i}-y_{j}\right)\right\},
$$

and

$$
\begin{aligned}
I_{c}(x, 0)= & \sum_{i \in C_{1}^{\prime}, \jmath \in C_{2}^{\prime}} V_{\imath j}\left(y_{\imath}-y_{\jmath}+x\right)+\sum_{\jmath \in C_{1}^{\prime}} V_{2 \jmath}\left(-x-y_{j}\right) \\
& +\sum_{\jmath \in C_{2}^{\prime}} V_{1 \jmath}\left(x-y_{j}\right)+V_{12}(x) .
\end{aligned}
$$

Since $f_{j}=O\left(h^{2}|y|\right)$, we have formally: $P_{e}(x, h)=P_{e}(x, 0)+O\left(h^{2}\right)$. It is therefore natural to expect that the leading terms of the eigenvalues of $P_{e}(x, h)$ are given by those of $P_{e}(x, 0)$. The following result justifies this intuition.

Proposition 3.1. Assume (3.1) and let $\lambda_{1}(x, 0), \ldots, \lambda_{m}(x, 0)$ be the $m$ eigenvalues of $P_{e}(x, 0)$ satisfying (2.16) with $\delta>0$ independent of $x \in \mathbf{R}^{n}$. Then, for $h$ sufficiently small, there exist exactly $m$ eigenvalues $\lambda_{1}(x, h), \ldots, \lambda_{m}(x, h)$ of $P_{e}(x, h)$ such that

$$
\lambda_{j}(x, h)=\lambda_{j}(x, 0)+O\left(h^{2}\right), \text { for } j=1, \ldots, m,
$$

uniformly in $x$. In particular (2.16) is satisfied by $\lambda_{1}(x, h), \ldots, \lambda_{m}(x, h)$, uniformly in $x \in \mathbf{R}^{n}$ and $h>0$. Here all eigenvalues are counted according to their multiplicity.

Proof. Let $\Gamma(x)$ be a family of complex contours such that

$$
\operatorname{dist}\left(\sigma\left(P_{e}(x, 0)\right), \Gamma(x)\right) \geq \delta / 2
$$


and $\lambda_{1}(x, 0), \ldots, \lambda_{m}(x, 0)$ are in the interior of $\Gamma(x)$. Then it follows from a perturbation argument in $h$ that the spectral projections

$$
\begin{aligned}
& \Pi(x, 0)=\frac{1}{2 \pi i} \oint_{\Gamma(x)}\left(z-P_{e}(x, 0)\right)^{-1} d z \\
& \Pi(x, h)=\frac{1}{2 \pi i} \oint_{\Gamma(x)}\left(z-P_{e}(x, h)\right)^{-1} d z
\end{aligned}
$$

are well defined if $h>0$ is sufficiently small. Making us of the exponential decay of eigenfunctions and the Taylor expansion in $f_{1}, f_{2}$ of the interaction $I_{c}(x, h)$ [defined in (2.6)], one obtains: $\left.\| P_{e}(x, h)-P_{e}(x, 0)\right) \Pi(x, 0) \| \leq C h^{2}$, uniformly in $x \in \mathbf{R}^{n}$. Consequently we derive from (3.2) that

$$
\|(\Pi(x, h)-\Pi(x, 0)) \Pi(x, 0)\| \leq C h^{2},
$$

uniformly in $x$. In the same way, we can prove that

$$
\|(\Pi(x, h)-\Pi(x, 0)) \Pi(x, h)\| \leq C h^{2},
$$

uniformly in $x \in \mathbf{R}^{n}$. Now the desired result follows from the arguments of [HS1]. See Proposition 1.4 and 2.6 in [HS1].

In the case $m=1$, we can show that $\lambda_{1}(x, 0)$ and $\lambda_{1}(x, h)$ are smooth and

$$
\left|\partial_{x}^{\alpha}\left(\lambda_{1}(x, h)-\lambda_{1}(x, 0)\right)\right|=O\left(h^{2}\langle x\rangle^{-\varrho}\right) \text { for any } \alpha \in \mathbf{N}^{n}, \alpha \neq 0 .
$$

Next, let $P^{A D}(h)$ denote the adiabatic Hamiltonian defined as before. Let $R^{A D}(z, h)=\left(P^{A D}(h)-z\right)^{-1}$ denote the resolvent of $P^{A D}(h)$. To estimate the boundary values of the resolvent, we use Mourre's commutator method. Recall that the notion of conjugate operator was introduced by Mourre in [M].

Theorem 3.2. Assume that $A$ is a conjugate operator of $P^{A D}(h)$ at $E \in \mathbf{R}$, which in addition satisfies

$$
\begin{aligned}
& i\left[P^{A D}(h), A\right] \geq C h>0, \quad \text { on } \quad \operatorname{Ran}\left(E_{I}\left(P^{A D}(h)\right)\right) \\
& \left\|\left[\left[P^{A D}(h), A\right], A\right]\left(P^{A D}(h)+i\right)^{-1}\right\| \leq C h^{2} .
\end{aligned}
$$

Here $I$ is a small interval around $E$, and $E_{I}(\cdot)$ denotes the spectral projection associated with I. Then one has

$$
\left\|\langle A\rangle^{-s} R^{A D}(\lambda \pm i 0, h)\langle A\rangle^{-s}\right\| \leq C h^{-1}
$$

for $s>1 / 2, \lambda$ sufficiently close to $E$ and $h>0$ sufficiently small.

Theorem 3.2 is just a parameter-dependent version of Mourre's result (see [GM, M]). We do not give the details of the proof here. To check the condition (3.5) we need some non-trapping assumption on the eigenvalue curves $\lambda(x, h)$ of $P_{e}(x, h)$ (see $[\mathrm{RT}])$. We shall say that a classical Hamiltonian $q$ is non-trapping at the energy $E$ if the classical trajectories satisfy:

$$
\lim _{|t| \rightarrow \infty}\left|e^{t H_{q}}(x, \xi)\right|=+\infty, \quad \forall(x, \xi) \in q^{-1}(E) .
$$

Here $H_{q}$ is the Hamiltonian vector field of $q$. In the case $q(x, \xi)=\xi^{2}+V(x)$ with $V$ a two-body potential tending to 0 at the infinity, the non-trapping assumption is satisfied at the energy $E>0$ if $2 E-2 V(x)-x \cdot \nabla V(x)>0$ for all $x$. 
Corollary 3.3. Assume that $\operatorname{rank} \Pi(x, h)=1$. Let $\lambda(x, h)$ and $\lambda(x, 0)$ denote the corresponding eigenvalue of $P_{e}(x, h)$ and $P_{e}(x, 0)$. Assume that the classical Hamiltonian $p(x, \xi)=\xi^{2}+\lambda(x, 0)$ is non-trapping at the energy $E \in \mathbf{R}$. Then there exists a conjugate operator $A$ of $P^{A D}(h)$ such that (3.5) is satisfied.

Proof. Making use of the non-trapping assumption, we can construct, by the method of [GM], a symbol $a(x, \xi)$ such that $a(x, \xi)-x \cdot \xi$ is a bounded symbol and

$$
\{p, a\} \geq C>0, \quad \text { for }(x, \xi) \in p^{-1}(I),
$$

where $I$ is a small interval around $E$ and $\{\cdot, \cdot\}$ denotes the Poisson bracket. Let $A=\Pi a^{w}(x, h D) \Pi$, where $a^{w}(x, h D)$ is the $h$-pseudodifferential operator with Weyl symbol $a(x, \xi)$ defined by:

$$
\left(a^{w}(x, h D) u\right)(x)=(2 \pi)^{-n} \iint e^{\imath(x-y) \cdot \xi} a((x+y) / 2, h \xi) u(y) d y d \xi,
$$

for $u \in Y$. Since $\Pi$ commutes with $A$ and with the multiplication by $\lambda(x, h)$, on can check that

$$
\begin{aligned}
i\left[P^{A D}, A\right] & =i\left[\Pi\left(-h^{2} \Delta_{x}\right) \Pi+\lambda(x, h) \Pi, A\right] \\
& =i \Pi\left[-h^{2} \Delta_{x}+\lambda(x, h), A\right] \Pi .
\end{aligned}
$$

To simplify notations, we write: $a^{w}=a^{w}(x, h D)$. Then

$$
\begin{aligned}
& i \Pi\left[-h^{2} \Delta_{x}+\lambda(x, h), A\right] \Pi \\
& \quad=i \Pi\left[-h^{2} \Delta_{x}+\lambda(x, h), a^{w}\right] \Pi+\Pi\left[-h^{2} \Delta, \Pi\right] a^{w} \Pi+\Pi a^{w}\left[-h^{2} \Delta, \Pi\right] \Pi .
\end{aligned}
$$

Making use of results on symbolic calculus of h-pseudo-differential operators (see [Ro]), we derive from the positivity of the Poisson bracket $\{p, a\}$ and the fact that $\lambda(x, h)=\lambda(x, 0)+O\left(h^{2}\right)$ the following lower bound on the first term in the above equality:

$$
i I I\left[-h^{2} \Delta_{x}+\lambda(x, h), a^{w}\right] \Pi \geq C h, \quad C>0
$$

for $h>0$ small enough, on the range of $E_{J}\left(P^{A D}(h)\right)$, where $J \subset I$ is a small interval containing $E$. To estimate the other two terms, we remark: $\left.\left[-h^{2} \Delta, \Pi\right]\right]=$ $-2 h \nabla \Pi \cdot\left(h D_{x}\right)+O\left(h^{2}\right)$, where $O\left(h^{2}\right)$ is a bounded operator with norm bounded by $C h^{2}$. Since $\Pi(\nabla \Pi) \Pi=0$ and $\left[I I, a^{w}\right]=O(h)$, we obtain:

$$
\Pi\left[-h^{2} \Delta, \Pi\right] a^{w} \Pi+\Pi a^{w}\left[-h^{2} \Delta, \Pi\right] \Pi=O\left(h^{2}\right) .
$$

This proves that $i\left[P^{A D}, A\right] \geq C^{\prime} h, C^{\prime}>0$, on the range of $E_{J}\left(P^{A D}\right)$, for $h>0$ small enough. The second estimate in (3.5) can be readily verified. $A$ is clearly a conjugate operator of $P^{A D}$. See the definition in [M].

We derive from Corollary 3.3 that for any $s>1 / 2$, one has

$$
\left\|\langle x\rangle^{-s} R^{A D}(\lambda \pm i 0, h)\langle x\rangle^{-s}\right\| \leq C h^{-1}, \quad \text { for } \lambda \text { near } E
$$

By the method of multiple commutators, we can derive as in [W2] the high order estimates, $\forall s>k+1 / 2$ :

$$
\left\|\langle x\rangle^{-s} \partial_{\lambda}^{k} R^{A D}(\lambda \pm i 0, h)\langle x\rangle^{-s}\right\| \leq C_{k} h^{-k-1},
$$

for $\lambda$ near $E$. This allows to obtain the decay estimates on semiclassical wave functions: $\forall s>0, \forall \varepsilon>0$, one has

$$
\left\|\langle x\rangle^{-s} f\left(P^{A D}(h)\right) e^{-i t P^{A D}(h) / h}\langle x\rangle^{-s}\right\| \leq C_{\varepsilon s} h^{-\varepsilon}\langle t\rangle^{-s+\varepsilon},
$$

for all $t \in \mathbf{R}$. 
Let now $e_{1}(h), \ldots, e_{k}(h)$ be the first $k$ points (counted from below) in $\sigma_{d}\left(P^{c}(h)\right.$ ) with total multiplicity $m$. Assume that:

$$
e_{1}(h), \ldots, e_{k}(h) \text { are all stable [cf. (2.9)] }
$$

Let $\lambda_{1}(x, h), \ldots, \lambda_{m}(x, h)$ be the first $m$ eigenvalues (counted with their multiplicity) of $P_{e}(x, h)$ with $\lim _{|x| \rightarrow \infty} \lambda_{\jmath}(x, h)=e_{j}(h)$ for some $1 \leq j \leq k$. Assume that

$$
\operatorname{dist}\left(\sigma\left(P_{e}(x, h)\right) \backslash\left\{\lambda_{1}(x, h), \ldots, \lambda_{m}(x, h)\right\},\left\{\lambda_{1}(x, h), \ldots, \lambda_{m}(x, h)\right\}\right) \geq \delta>0,
$$

uniformly in $x$ and $h>0$. In view of Proposition 3.1, this just requires (2.16) to be satisfied by $\lambda_{1}(x, 0), \ldots, \lambda_{m}(x, 0)$.

Theorem 3.4. Assume the conditions (3.1), (3.11), and (3.12). Let $\Pi(x, h)$ be the spectral projection associated to the first $m$ eigenvalues $\lambda_{1}(x, h), \ldots, \lambda_{m}(x, h)$ of $P_{e}(x, h)$. Let $P^{A D}(h)$ be the adiabatic part of $P(h)$ defined as before. Let $\lambda_{0} \in \mathbf{R}$ be such that

$$
\lambda_{0}<\inf _{x} \inf \left(\sigma\left(P_{e}(x, h)\right) \backslash\left\{\lambda_{1}(x, h), \ldots, \lambda_{m}(x, h)\right\}\right),
$$

and that for $\left|\lambda-\lambda_{0}\right|$ sufficiently small

$$
\left\|\langle x\rangle^{-s} R^{A D}(\lambda \pm i 0, h)\langle x\rangle^{-s}\right\| \leq C h^{-1}, \quad \frac{1}{2}<s<\frac{\varrho}{2}
$$

Then we have

$$
\left\|\langle x\rangle^{-s} R(\lambda \pm i 0, h)\langle x\rangle^{-s}\right\| \leq C h^{-1} .
$$

Here $R(\lambda \pm i 0, h)=\lim _{\varepsilon \downarrow 0}(P-(\lambda \pm i \varepsilon))^{-1}$ denote the boundary values of the resolvent of $P$, and $s$ and $\lambda$ are as in (3.14).

Proof. Recalling (1.9) and setting

$$
V=P-P^{A D}-Q^{A D}
$$

and

$$
R^{A D}(z, h)=\left(P^{A D}(h)-z\right)^{-1} \Pi, \quad \widehat{R}(z, h)=\left(Q^{A D}-z\right)^{-1} \widehat{\Pi},
$$

one finds the following identity

$$
R(z, h)=R^{A D}(z, h)+\widehat{R}(z, h)-R(z, h)\left(\widehat{\Pi} P R^{A D}(z, h)+\Pi P \widehat{R}(z, h)\right) .
$$

Applying this equality once more, we obtain:

$$
\begin{aligned}
R(z, h)= & R^{A D}(z, h)+\widehat{R}(z, h)-\left(\widehat{R}(z, h) P R^{A D}(z, h)+R^{A D}(z, h) P \widehat{R}(z, h)\right) \\
& +R(z, h)\left(\widehat{\Pi P} P R^{A D}(z, h) P \widehat{R}(z, h)+\Pi P \widehat{R}(z, h) P R^{A D}(z, h)\right)
\end{aligned}
$$

for $z=\lambda+i \varepsilon, \varepsilon \neq 0$. Note that (3.13) implies

$$
\|\widehat{R}(z, h)\| \leq C, \quad \text { uniformly in } h>0, \lambda \text { near } \lambda_{0} \text { and } \varepsilon \in[-1,1] .
$$

Since

$$
\widehat{\Pi} P \Pi=-h^{2} \widehat{\Pi} \Delta_{x} \Pi=h O\left(\langle x\rangle^{-\varrho}\right) h D_{x}+h^{2} O\left(\langle x\rangle^{-\varrho}\right)
$$

on $\operatorname{Ran} \Pi(x, h)$, we obtain

$$
\left\|\langle x\rangle^{s}\left(\widehat{\Pi} P R^{A D}(z, h) P \widehat{R}(z, h)+\Pi P \widehat{R}(z, h) P R^{A D}(z, h)\right)\langle x\rangle^{-s}\right\| \leq C h,
$$


uniformly in $z=\lambda+i \varepsilon$ with $\lambda$ and $\varepsilon$ as in (3.18). This shows that for $h>0$ sufficiently small, one has an expression for $R(z, h)$ in terms of $R^{A D}(z, h)$ and $\widehat{R}(z, h)$ :

$$
\begin{aligned}
R(z, h)= & \left(R^{A D}(z, h)+\widehat{R}(z, h)-\left(A(z, h)+A(\bar{z}, h)^{*}\right)\right) \\
& \times(1-B(z, h))^{-1}, \quad \text { for } \varepsilon \neq 0,
\end{aligned}
$$

where

$$
A(z, h)=\widehat{R}(z, h) P R^{A D}(z, h), \quad B(z, h)=\widehat{\Pi} P A(z, h)^{*}+\Pi P A(z, h) .
$$

The estimate (3.15) follows easily.

We remark that according to Corollary 3.3., the estimate (3.15) on the boundary values of the resolvent holds if the first eigenvalue of $P_{e}(x, 0)$ is non-trapping at the energy

$$
\lambda_{0}<\inf _{x} \inf \left(\sigma\left(P_{e}(x, 0)\right) \backslash\left\{\lambda_{1}(x, 0)\right\}\right) .
$$

It is however clear that Theorem 3.4 applies to more general situations. Assume for instance that there exist globally defined smooth sections of eigenfunctions $u_{j}(x, h)$ with

$$
P_{e}(x, h) u_{j}(x, h)=\lambda_{\jmath}(x, h) u_{\jmath}(x, h), \quad j=1,2, \ldots, m .
$$

Then $P^{A D}(h)$ can be smoothly diagonalized up to the order $O(h)$. Assume that for $p_{j} \equiv \xi^{2}+\lambda_{j}(x, 0), j=1, \ldots, m$, we can construct a same symbol a such that $\left\{p_{j}, a\right\} \geq C>0$, for $(x, \xi) \in p_{j}^{-1}\left(\lambda_{0}\right), j=1, \ldots, m$. Then we can construct a conjugate operator $A$ so that (3.5) holds. In this case, (3.15) is still true. Equation (3.14) is a kind of non-trapping condition on the effective Hamiltonians $\xi^{2}+\lambda_{1}(x, 0), \ldots, \xi^{2}+\lambda_{m}(x, 0)$. Theorem 3.4 can be interpreted as follows: if the local energy of the adiabatic dynamics $e^{-i t P^{A D} / h}$ has a uniform (in $h>0$ ) timedecay, so does the total dynamics $e^{-i t P / h}$. See Lemma 4.2 and also [W2] for two-body problems.

\section{Born-Oppenheimer Approximation of Wave Operators}

The purpose of this section is to show the usefulness of the Born-Oppenheimer approximation: Under the assumptions of Theorem 3.4, the adiabatic wave operators $\Omega_{ \pm}^{A D}$ are good approximations of the cluster wave operators $\Omega_{ \pm}^{c}$. Let us first study the non-adiabatic wave operators $\Omega_{ \pm}^{N A D}$. Setting

$$
U(t, h)=e^{-i t P / h}, \quad U_{A D}(t, h)=e^{-i t P^{A D} / h}
$$

and denoting by $E_{\mathrm{ac}}\left(P^{A D}\right)$ the projection onto the absolutely continuous spectral subspace of $P^{A D}$, we have

$$
\Omega_{ \pm}^{N A D}(h)=\mathrm{s}-\lim _{t \rightarrow \pm \infty} U(t, h)^{*} U_{A D}(t, h) E_{\mathrm{ac}}\left(P^{A D}\right) .
$$

The main result of this section is the following

Theorem 4.1. Let $\lambda_{0}, P^{A D}$ be the same as in Theorem 3.4. Let $\chi \in C_{0}^{\infty}(\mathbf{R})$ with support sufficiently near $\lambda_{0}$, such that $Q^{A D}(x)>\sup \operatorname{supp} \chi$, on $R(\widehat{\Pi}(x, h))$ for all $x \in \mathbf{R}^{n}$. Then, under the assumptions of Theorem 3.4, we have

$$
\left\|\left(\Omega_{ \pm}^{N A D}(h)-1\right) \chi\left(P^{A D}\right)\right\|=O(h), \quad \text { as } h \rightarrow 0 .
$$

Here $\|$.$\| denotes the norm of bounded operators on L^{2}\left(\mathbf{R}^{n(N+1)}\right)$. 
Remark. In Theorem 4.1, we use, among other things, the assumption (3.14) which is a kind of non-trapping condition. This condition is used to establish the uniform time-decay: (4.3) and (4.4). For semiclassical N-body Schrödinger operators, it is proven in Sect. 4 of [W4] that an estimate of the form (4.4) uniform in $h>0$ implies that the classical Hamiltonian and all classical sub-Hamiltonians are non-trapping at energy $E, \forall E \in \operatorname{supp} \phi$. This leads us to believe that a non-trapping condition is necessary in establishing (4.2).

To prove Theorem 4.1, we need some preparations.

Lemma 4.2. Assume that for some $s>1 / 2$ and $\delta>0$,

$$
\left\|\langle x\rangle^{-s} R^{A D}(\lambda \pm i 0, h)\langle x\rangle^{-s}\right\| \leq C h^{-1}, \text { for } \lambda \in\left(\lambda_{0}-\delta, \lambda_{0}+\delta\right) .
$$

Then, for any $\phi \in C_{0}^{\infty}\left(\left(\lambda_{0}-\delta, \lambda_{0}+\delta\right)\right)$, we have

$$
\int_{-\infty}^{+\infty}\left\|\langle x\rangle^{-s} U_{A D}(t, h) \phi\left(P^{A D}\right) f\right\|^{2} d t \leq C\|f\|^{2},
$$

and if $\lambda_{0}$ satisfies the assumptions of Theorem 3.4,

$$
\int_{-\infty}^{+\infty}\left\|\langle x\rangle^{-s} U(t, h) \phi(P) f\right\|^{2} d t \leq C\|f\|^{2},
$$

for all $f \in L^{2}\left(\mathbf{R}^{n(N+1)}\right)$, uniformly in $h \in\left(0, h_{0}\right)$.

Proof. By Theorem 3.4, $\langle x\rangle^{-s}$ is locally $P^{A D}$ - and $P$-smooth. The results (in particular, the uniformity in $h>0$ ) follow from the arguments used in the proof of Lemma 2.1 in [W2].

Lemma 4.3. Let $\chi \in C_{0}^{\infty}((a, b))$. Assume $b<\inf \sigma\left(Q^{A D}\right)$. Then we have

$$
\left\|\left(\chi(P)-\chi\left(P^{A D}\right)\right)\langle x\rangle^{\varrho}\right\| \leq C h .
$$

Proof. For any self-adjoint operator $A$ and any real function $\chi \in C_{0}^{\infty}(\mathbf{R})$, one has the formula (see Eq. (13) in [HS2]):

$$
\chi(A)=\frac{1}{\pi} \int \frac{\partial \tilde{\chi}}{\partial \bar{z}}(z)(A-z)^{-1} L(d z)
$$

where $L(d z)$ is the Lebesgue measure over $\mathbf{C}, \tilde{\chi}$ denotes an almost holomorphic extension of $\chi$ with compact support, i.e.

(i) $\tilde{\chi} \in C_{0}^{\infty}(\mathbf{C})$ with $\tilde{\chi}(x)=\chi(x)$, for $x \in \mathbf{R}$.

(ii) $\partial_{\bar{z}} \tilde{\chi}(z)=O\left(|\operatorname{Im} z|^{\infty}\right)$.

Admitting the existence of such an almost holomorphic extension (see the references in [HS2]), the reader can check the formula (4.6) by using Stone's formula and an integration by parts in $\bar{z}$ variable. Making use of (3.17), we get

$$
R(z)=R^{A D}(z)+\widehat{R}(z)+B(z),
$$

where the remainder term $B(z)$ satisfies the estimate

$$
\left\|B(z)\langle x\rangle^{\varrho}\right\| \leq C h|\operatorname{Im} z|^{-N_{0}} \quad \text { for } \quad z \in \operatorname{supp} \tilde{\chi}, \operatorname{Im} z \neq 0,
$$


with $N_{0} \in \mathbf{N}$ being independent of $z$ and $h$. Here we just used the self-adjointness of $P(h)$ and $P^{A D}(h)$ to conclude that

$\left\|\langle x\rangle^{\varrho} R(z)\langle x\rangle^{-\varrho}\right\| \leq C|\operatorname{Im} z|^{-N_{0}^{\prime}}, \quad\left\|\langle x\rangle^{\varrho} R^{A D}(z)\langle x\rangle^{-\varrho}\right\| \leq C|\operatorname{Im} z|^{-N_{0}^{\prime}}, \quad \operatorname{Im} z \neq 0$,

for some $N_{0}^{\prime}$ depending only on $\varrho$. Since $\widehat{R}(z)$ is holomorphic on the support of $\tilde{\chi}$ for an appropriate choice of $\tilde{\chi}$, Lemma 4.3 follows easily.

Now we are ready to give the

Proof of Theorem 4.1. Under the assumptions of Theorem 4.1, we can choose $\delta>0$ such that $\lambda_{0}+\delta<\inf \sigma\left(Q^{A D}\right)$ and the resolvent estimate (3.15) of Theorem 3.4 is valid on the interval $\left[\lambda_{0}-\delta, \lambda_{0}+\delta\right]$. Let $\chi \in C_{0}^{\infty}\left(\left(\lambda_{0}-\delta, \lambda_{0}+\delta\right)\right)$. Take $\phi \in C_{0}^{\infty}\left(\left(\lambda_{0}-\delta, \lambda_{0}+\delta\right)\right)$ with $\phi(\lambda)=1$ on supp $\chi$. By the intertwining property of wave operators,

$$
\phi(P) \Omega_{ \pm}^{N A D} \chi\left(P^{A D}\right)=\Omega_{ \pm}^{N A D} \chi\left(P^{A D}\right) .
$$

This allows us to derive:

$$
\begin{aligned}
\left(\Omega_{+}^{N A D}-1\right) \chi\left(P^{A D}\right)= & \frac{i}{h} \int_{0}^{+\infty} \phi(P) U(t, h)^{*} V U_{A D}(t, h) \chi\left(P^{A D}\right) d t \\
& +(\phi(P)-1) \chi\left(P^{A D}\right) .
\end{aligned}
$$

According to Lemma 4.3 one has

$$
(\phi(P)-1) \chi\left(P^{A D}\right)=\left(\phi\left(P^{A D}\right)-1\right) \chi\left(P^{A D}\right)+O(h)=O(h)
$$

in the space of bounded linear operators $\mathscr{C}\left(L^{2}\left(\mathbf{R}^{n(N+1)}\right)\right)$. To estimate the first term of the rhs of (4.7), we recall that

$$
\begin{aligned}
V & =-h^{2}\left(\Pi \Delta_{x} \widehat{\Pi}+\widehat{\Pi} \Delta_{x} \Pi\right) \\
& =-2 h \widehat{\Pi}\left(\partial_{x} \Pi\right) h \nabla_{x}+O\left(h^{2}\langle x\rangle^{-\varrho}\right),
\end{aligned}
$$

on $\operatorname{Ran} \Pi$. Take $\psi \in C_{0}^{\infty}\left(\left(\lambda_{0}-\delta, \lambda_{0}+\delta\right)\right)$ with $\psi(\lambda)=1$ on supp $\phi$. Combining (4.8) with Lemma 4.3, we get

$$
\begin{aligned}
\psi(P) V & =-2 \psi\left(P^{A D}\right) h \widehat{\Pi} \partial_{x} \Pi h \nabla_{x}+O\left(h^{2}\langle x\rangle^{-\varrho}\right) \\
& =O\left(h^{2}\langle x\rangle^{-\varrho}\right), \quad \text { on } \operatorname{Ran} \Pi,
\end{aligned}
$$

since $\psi\left(P^{A D}\right) \widehat{\Pi}=\psi(0) \widehat{\Pi}=0$. Applying Lemma 4.2, we see that

$$
\begin{aligned}
& \left|\left\langle\int_{0}^{+\infty} \phi(P) U(t, h)^{*} V U_{A D}(t, h) \chi\left(P^{A D}\right) u d t, v\right\rangle\right| \\
& \quad \leq C\left\|\langle x\rangle^{\varrho / 2} \psi(P) V\langle x\rangle^{\varrho / 2}\right\|\|u\|\|v\| \leq C^{\prime} h^{2}\|u\|\|v\|,
\end{aligned}
$$

forall $u, v \in L^{2}$. Therefore we can conclude that

$$
\frac{i}{h} \int_{0}^{+\infty} \phi(P) U(t, h)^{*} V U_{A D}(t, h) \chi\left(P^{A D}\right) d t
$$


defines a bounded operator with norm bounded by $C h$. Equation (4.2) for $\Omega_{+}^{N A D}$ follows from (4.7). Using the same arguments, we can obtain a similar bound for the operator $\left(\Omega_{-}^{N A D}(h)-1\right) \chi\left(P^{A D}\right)$.

As a consequence of Theorem 4.1, we obtain the following

Corollary 4.4. Let $\lambda_{1}(x, h)$ be the first eigenvalue of $P_{e}(x)$. Denote by $P^{A D}$ the adiabatic approximation of $P$ associated to $\lambda_{1}(x, h)$. Let $\lambda_{0} \in \mathbf{R}$ with $\lambda_{0}<$ $\inf \sigma\left(Q^{A D}\right)$. Assume that $\lambda_{0}$ is non-trapping for $p(x, \xi)=\xi^{2}+\lambda_{1}(x, 0)$. Then there exists $\delta>0$ such that for any $\chi \in C_{0}^{\infty}\left(\left(\lambda_{0}-\delta, \lambda_{0}+\delta\right)\right)$ we have

$$
\left\|\left(\Omega_{ \pm}^{N A D}(h)-1\right) \chi\left(P^{A D}\right)\right\| \leq C_{\chi} h .
$$

For the cluster wave operators $\Omega_{ \pm}^{c}$ we have according to Theorem 2.3

$$
\Omega_{ \pm}^{c} \Pi_{0}=\Omega_{ \pm}^{N A D} \Omega_{ \pm}^{A D} .
$$

Theorem 4.1 gives the adiabatic approximation of the wave operator $\Omega_{ \pm}^{c}$ up to order $O(h)$.

Corollary 4.5. Under the assumptions of Theorem 3.4, there exists $\delta>0$ such that for any $\chi \in C_{0}^{\infty}\left(\left(\lambda_{0}-\delta, \lambda_{0}+\delta\right)\right)$ one has

$$
\left.\| \Omega_{ \pm}^{c}-\Omega_{ \pm}^{A D}\right) \chi\left(P_{c}\right) \Pi_{0} \| \leq C_{\chi} h .
$$

Proof. It suffices to use the intertwining relation of the wave operators and the results of Theorem 4.1. Take $\phi \in C_{0}^{\infty}\left(\left(\lambda_{0}-\delta, \lambda_{0}+\delta\right)\right)$ with $\phi(\lambda)=1$ on supp $\chi$. Then

$$
\Omega_{ \pm}^{c} \chi\left(P^{c}\right) \Pi_{0}=\Omega_{ \pm}^{N A D} \phi\left(P^{A D}\right) \Omega_{ \pm}^{A D} \chi\left(P^{c}\right) \Pi_{0}=\Omega_{ \pm}^{A D} \chi\left(P^{c}\right) \Pi_{0}+O(h)
$$

in $\mathscr{C}\left(L^{2}\left(\mathbf{R}^{n(N+1)}\right)\right.$, by Theorem 4.1 .

\section{The Classical Limit of the Adiabatic Wave Operators}

It is a well known principle in the physics literature that the dynamics of heavy particles in molecular collisions can be well approximated by classical mechanics. In this section we shall verify this assertion in studying the classical limit of the adiabatic wave operators $\Omega_{ \pm}^{A D}(h)$. Our approach is parallel to [W3]: Since $P^{A D}$ essentially is a two-body Hamiltonian, we can construct a good approximation of $\Omega_{ \pm}^{A D}(h)$ by use of Fourier integral operators. The new point now is the existence of a spectral projection, $\Pi(x, h)$, which enters into the leading term of the amplitude. In the following we shall concentrate on clarifying the leading terms of various approximations and only skip over the details of the remainder estimates which are often the same as in [W3]. Note that the similar results to Theorem 5.3 for the classical limit of wave operators for usual two-body Schrödinger operators have not been explicitly stated in the literature. See however a remark in the Introduction of [W3] and also [Y] in other framework.

Let $E_{0}$ be a simple eigenvalue of $P^{c} . E_{0}$ is $h$-dependent, but assuming (3.1) one can easily verify that $E_{0}(h) \equiv E_{o}$ has an asymptotic expansion

$$
E_{0}(h) \sim \sum_{j=0}^{\infty} h^{2 j} e_{j} .
$$


Assume that $E_{0}(h)$ is stable (see (2.9)). Let $\lambda_{1}(x, h)$ be the eigenvalue of $P_{e}(x, h)$ such that

$$
\lambda_{1}(x, h)-E_{0}(h)=O\left(\langle x\rangle^{-\varrho}\right), \quad \text { as } \quad|x| \rightarrow \infty,
$$

with $\varrho>1$ as in (3.1). Assume that

$$
\operatorname{dist}\left(\lambda_{1}(x, h), \sigma\left(P_{e}(x, h)\right) \backslash\left\{\lambda_{1}(x, h)\right\}\right) \geq \delta>0,
$$

uniformly in $x \in \mathbf{R}^{n}$ and $h>0$. Under the assumption (3.1) we can show that $\lambda_{1}(x, h)$ possesses an asymptotic expansion

$$
\lambda_{1}(x, h) \sim \sum_{j=0}^{\infty} h^{2 \jmath} \Lambda_{j}(x)
$$

uniformly in $x$. In particular, $\Lambda_{0}(x)$ is an eigenvalue of $P_{e}(x, 0)$ (in fact $\Lambda_{0}(x)=$ $\lambda_{1}(x, 0)$, see Proposition 3.1) and one has:

$$
\left|\Lambda_{\jmath}(x)-e_{\jmath}\right| \leq C_{\jmath}\langle x\rangle^{-\varrho} .
$$

We want to study the relation between $\Omega_{ \pm}^{A D}(h)$ and the classical wave operators

$$
\Omega_{ \pm}^{\mathrm{cl}}(x, \xi)=\lim _{t \rightarrow \pm \infty} \Phi^{-t} \circ \Phi_{0}^{t}(x, \xi), \quad \xi \neq 0
$$

where $\Phi^{t}$ and $\Phi_{0}^{t}$ denote the classical Hamiltonian flows associated to $p(x, \xi)=$ $\xi^{2}+\Lambda_{0}(x)-e_{0}$ and $p_{0}(x, \xi)=\xi^{2}$. We shall only study the outgoing wave operator $\Omega^{A D}(h)=\Omega_{+}^{A D}(h)$.

Given a phase $\phi(.,$.$) , we denote by J(a)$ the Fourier integral operator defined by:

$$
J(a) f(x)=(2 \pi h)^{-n} \int e^{i\left(\phi(x, \xi)-x^{\prime} \cdot \xi\right) / h} a(x, \xi ; h) f\left(x^{\prime}\right) d x^{\prime} d \xi
$$

with $a \in C^{\infty}\left(\mathbf{R}_{x, \xi}^{2 n}, \mathscr{L}\left(L^{2}\left(\mathbf{R}_{y}^{n N}\right)\right)\right.$ with bounded derivatives and $f$ in the Schwartz space $\mathscr{f}\left(\mathbf{R}_{x}^{n}, L^{2}\left(\mathbf{R}_{y}^{n N}\right)\right)$. We refer the reader to Appendix in [W1] for results on the calculus and continuity of this class of Fourier integral operators but with scalar amplitude $a$. When $a$ is $\mathscr{L}\left(L^{2}\right)$-valued, we can establish the same results in replacing $|a|$ by $\|a\|$ in the proofs.

We want to construct $\phi$ and a so that we can compare $U_{A D}(t, h)$ with $J(a) U_{0}(t, h) \Pi_{0}(h)$ for $t \rightarrow+\infty$. Here $\Pi_{0}(h)$ is the spectral problem of $P^{c}(h)$ associated to $E_{0}(h)$ and the free evolution is

$$
U_{0}(t, h)=e^{-i t\left(-h^{2} \Delta_{x}+E_{0}\right) / h} .
$$

As usual, we determine the phase function $\phi$ and the amplitude $a$ by considering the formal identity

$$
\Pi(x, h)\left(-h^{2} \Delta_{x} \Pi(x, h)+\lambda_{1}(x, h)\right)\left(e^{\imath \phi / h} a(h)\right)=e^{i \phi(x, \xi) / h} a(h)\left(\xi^{2}+E_{0}(h)\right)
$$

and requiring that $\phi(x, \xi)-x \cdot \xi$ is small when $x \rightarrow \infty$. We look for $a(x, \xi ; h)$ in the form:

$$
a(x, \xi ; h)=\Pi(x, h) b(x, \xi ; h)
$$

with $b(h)$ satisfying

$$
\left(-h^{2} \Delta_{x}-h^{2}\left[\Delta_{x}, \Pi(x, h)\right]+\lambda_{1}(x, h)-\left(\xi^{2}+E_{0}(h)\right)\right) e^{i \phi / h} b(h)=0 .
$$


To determine $b(h) \sim \sum h^{j} b_{j}$, we expand $\Pi(x, h), \lambda_{1}(x, h)$ and $E_{0}(h)$ in powers of (h):

$$
\begin{aligned}
\Pi(x, h) & \sim \sum_{j=0}^{\infty} h^{2 \jmath} \pi_{j}(x), \\
\lambda_{1}(x, h) & \sim \sum_{j=0}^{\infty} h^{2 \jmath} \Lambda_{j}(x), \\
E_{0} & \sim \sum_{\jmath=0}^{\infty} h^{2 \jmath} e_{j} .
\end{aligned}
$$

Substituting these expressions into (5.8) and setting the resulting coefficient of $h^{k}$ equal to 0 for $k \in \mathbf{N}$, we obtain the following equations

$$
\begin{aligned}
\left|\nabla_{x} \phi(x, \xi)\right|^{2}+\Lambda_{0}(x)-e_{0}-\xi^{2} & =0, \\
\left(2 \nabla_{x} \phi \cdot \nabla_{x}+2 \nabla_{x} \phi \cdot \nabla_{x} \pi_{0}(x)+\Delta_{x} \phi\right) b_{0} & =0, \\
\left(2 \nabla_{x} \phi \cdot \nabla_{x}+2 \nabla_{x} \phi \cdot \nabla_{x} \pi_{0}(x)+\Delta_{x} \phi\right) b_{k} & =f_{k}, \quad k \geq 1,
\end{aligned}
$$

where $f_{k}$ depends only on the amplitudes $b_{j}$ with $0 \leq j \leq k-1$.

Since $\Lambda_{0}(x)-e_{0}$ is short-range, we can use the phase function $\phi$ constructed in [W3] to solve the eikonal equation (5.9) in the outgoing region

$$
\Omega(\varepsilon, d, R)=\left\{(x, \xi) \in R^{2 n} ; x \cdot \xi \geq(-1+\varepsilon)|x||\xi|,|\xi|>d,|x|>R\right\} .
$$

Here $\varepsilon, d>0$ are arbitrary, while $R=R(\varepsilon, d)$ should be large enough. Note that this phase function differs from that constructed by Isozaki-Kitada in [IK] by a function of $\xi$. It has the advantage of being related to the classical wave operators in an explicit way: It is a generating function for the classical wave operator acting as a canonical map on phase space (see [W3] and Eq. (5.25) below). In particular, one has

$$
\left|\partial_{x}^{\alpha} \partial_{\xi}^{\beta}(\phi(x, \xi)-x \cdot \xi)\right| \leq C_{\alpha \beta} R^{-\delta_{1}}\langle x\rangle^{-\delta_{2}-|\alpha|},
$$

for any $\delta_{1}, \delta_{2}>0$ with $\delta_{1}+\delta_{2}=\varrho-1$.

To construct a solution of (5.10), we use the method of characteristics. Let $\varrho_{1}(t ; x, \xi)$ denote the gradient flow of $\phi(x, \xi)$ :

$$
\frac{d}{d t} \varrho_{1}(t ; x, \xi)=\nabla \phi\left(\varrho_{1}(t ; x, \xi), \xi\right), \quad \varrho_{1}(0 ; x, \xi)=x .
$$

Here $\xi$ is considered as a parameter. For the properties of $\varrho_{1}(t ; x, \xi)$, we refer to Sect. 2 in [W1]. Put

$$
b_{0}=e^{F(x, \xi)} G_{0}, \quad F(x, \xi)=\frac{1}{2} \int_{0}^{\infty}\left(\Delta_{x} \phi\right)\left(\varrho_{1}(t ; x, \xi), \xi\right) d t
$$

where $G_{0}$ is to be determined by solving

$$
\nabla_{x} \phi \cdot \nabla_{x} G_{0}(x, \xi)+\left(\nabla_{x} \pi_{0}(x) \cdot \nabla_{x} \phi\right) G_{0}(x, \xi)=0 .
$$

Let $\pi_{00}$ denote the spectral projector of $P^{c}(0)$ associated with $e_{0}$. Then we have (see Theorem 2.2):

$$
\left\|\partial^{\alpha}\left(\pi_{0}(x)-\pi_{00}\right)\right\|=O\left(\langle x\rangle^{-\varrho-|\alpha|}\right) .
$$


Using the outgoing properties of the flow $\varrho_{1}(t ; x, \xi)$ (see [W1]), we obtain:

$$
\left\|\left(\nabla_{x} \pi_{0}\right) \varrho_{1}(t ; x, \xi)\right\| \leq C\langle t+|x|\rangle^{-\varrho-1},
$$

for $(x, \xi) \in \Omega(\varepsilon, d, R)$. Put

$$
G_{0}^{t}=G_{0}\left(\varrho_{1}(t ; x, \xi), \xi\right), \quad \pi^{t}=\frac{d}{d t} \pi_{0}\left(\varrho_{1}(t ; x, \xi)\right) .
$$

Along the flow $\varrho_{1}(t ; x, \xi), \mathrm{Eq} .(5.14)$ is transformed into an ordinary differential equation:

$$
\frac{d}{d t} G_{0}^{t}+\pi^{t} G_{0}^{t}=0
$$

Adding the condition

$$
\lim _{t \rightarrow \infty}\left\|G_{0}^{t}-I\right\|=0
$$

this equation can be solved by a series of Dyson:

$$
G_{0}^{t}=I+\int_{t}^{\infty} \pi^{t_{1}} d t_{1}+\cdots+\int_{t}^{\infty} \int_{t_{1}}^{\infty} \cdots \int_{t_{n-1}}^{\infty} \pi^{t_{1}} \cdots \pi^{t_{n}} d t_{n} \cdots d t_{1}+\cdots
$$

It can be checked that the norm of the general term in the above series is bounded by:

$$
C^{n} \int_{t}^{\infty} \int_{t_{1}}^{\infty} \cdots \int_{t_{n-1}}^{\infty}\left\langle t_{1}+|x|\right\rangle^{-\varrho-1} \cdots\left\langle t_{n}+|x|\right\rangle^{-\varrho-1} d t_{n} \cdots d t_{1}, \quad t>0
$$

for $(x, \xi) \in \Omega(\varepsilon, d, R)$. This shows that the series of Dyson is norm-convergent if $|x|>R$ with $R$ large enough. Setting now

$$
G_{0}(x, \xi)=G_{0}^{0}(x, \xi)
$$

one can verify that $G_{0}(\cdot, \cdot)$ is a smooth operator-valued solution of $(5.14)$ and

$$
\left\|G_{0}(x, \xi)-I\right\| \leq C\langle x\rangle^{1-\varrho}
$$

in any outgoing region $\Omega\left(\varepsilon^{\prime}, d^{\prime}, R^{\prime}\right)$ with $\varepsilon^{\prime}>\varepsilon, d^{\prime}>d, R^{\prime}>R$. The higher order transport equations (5.11), $k \geq 1$, can be solved by using the same arguments and an induction on $k$. Introducing a suitable cut-off $\chi$ and taking a $C^{\infty}$-realization of the amplitude

$$
a(x, \xi ; h) \sim \chi \Pi(x, h) \sum_{j \geq 0} h^{\jmath} b_{j}(x, \xi),
$$

we obtain a temporally global approximation of $U_{A D}(t, h)$ in outgoing regions.

Proposition 5.1. For any $d>0$, let $a(h), \phi$ be constructed as above. The we have

$$
\Omega^{A D}(h)=\mathrm{s}-\lim _{t \rightarrow \infty} U_{A D}(t, h)^{*} J(a) U_{0}(t, h) \Pi_{0}
$$

on $\operatorname{Ran} E_{2 d}\left(P_{0}(h)\right)$. Here $P_{0}(h)=-h^{2} \Delta_{x}$ and $E_{2 d}(\cdot)$ denotes the spectral projection onto the interval $(2 d,+\infty)$. 
Proof. It suffices to show that

$$
s-\lim _{t \rightarrow \infty} U_{0}(t, h)^{*} J(a) U_{0}(t, h)=\Pi_{0} \quad \text { on } \quad \operatorname{Ran} E_{2 d}\left(P_{0}(h)\right) .
$$

Since $J(a)$ can be regarded as a pseudo-differential operator with symbol $e^{-\imath(\phi-x \cdot \xi) / h} a$, this can be proven as in [W3] by making use of an Egorov's Theorem and the estimates (5.13), (5.14), and (5.15). The details are omitted (see [W3]).

By Proposition 5.1, we have, at least formally

$$
\Omega^{A D}(h)=W(t, h)+\frac{i}{h} \int_{t}^{\infty} U_{A D}(s, h)^{*} J(r) U_{0}(s, h) \Pi_{0} d s,
$$

where

$$
W(t, h)=U_{A D}(t, h)^{*} J(a) U_{0}(t, h) \Pi_{0}
$$

and $J(r)$ is defined as in (5.7) with amplitude given by

$$
r(h)=e^{-\imath \phi / h} P^{A D}\left(e^{\imath \phi / h} a(h)\right)-a(h)\left(\xi^{2}+E_{0}(h)\right) .
$$

Due to the choice of $a(h)$, we see that

$$
\left\|\partial_{x}^{\alpha} \partial_{\xi}^{\beta} r(x, \xi ; h)\right\| \leq C_{M \alpha \beta} h^{M}\langle x\rangle^{-M}, \text { for any } \quad M>1,
$$

for $(x, \xi) \in \Omega(2 \varepsilon, 2 d, 2 R)$. To give a meaning to the formal identity (5.18), we establish the following

Proposition 5.2. Let

$$
\begin{aligned}
S_{+}= & \left\{b \in C^{\infty}\left(\mathbf{R}^{2 n}\right) ; b \text { is a bounded symbol with } \operatorname{supp} b \subset \Omega(\varepsilon, d, R)\right. \\
& \text { for some } \varepsilon>0, d>0, R>0\} .
\end{aligned}
$$

Let $J(a)$ be a parametrix constructed as above such that the estimate (5.19) holds in the region $\Omega(\varepsilon / 2, d / 2, R / 2)$. Then, for any $b \in S_{+}$, with $\operatorname{supp} b \subset \Omega(\varepsilon, d, R)$, there is $T>0$ such that

$$
\left\|\left(\Omega^{A D}(h)-W(t, h)\right) b(x, h D)\right\| \leq C_{M} h^{M}\langle t\rangle^{-M}
$$

for all $M \in \mathbf{N}, t>T$. Furthermore, the estimate (5.20) is also true if we replace $b \in S_{+}$by $b_{0} \in C_{0}^{\infty}\left(\mathbf{R}_{x}^{n} \times\left(\mathbf{R}_{\xi}^{n} \backslash\{0\}\right)\right)$.

Proof. We use the identity (5.18) combined with microlocalized decay estimates on the free evolution of $U_{0}(t, h)$ (see [W3, Corollary 2.2]).

According to Proposition 5.2, to study the semiclassical approximation of $\Omega^{A D}(h)$, we only need to study $W(t, h)$ for a fixed $t>T$. Recall that in Proposition 5.2, no non-trapping condition on the effective Hamiltonian is needed, because we only used estimates on the free evolution $U_{0}(t, h)$ in the proof.

Now let $S=S_{+} \cup C_{0}^{\infty}\left(\mathbf{R}_{x}^{n} \times\left(\mathbf{R}_{\xi}^{n} \backslash\{0\}\right)\right)$. To relate $\Omega^{A D}(h)$ to the classical wave operators $\Omega^{\text {cl }}$ in the limit $h \rightarrow 0$, we shall study the action of $\Omega^{A D}$ on quantum observables. To this end we first observe that, using perturbation theory, one can show that $\Pi_{0}(h)$ possesses an asymptotic expansion

$$
\Pi_{0}(h) \sim \sum_{j=0}^{\infty} h^{2 j} \pi_{0 j}
$$


where $\pi_{0 j} \in \mathscr{L}\left(L^{2}\left(\mathbf{R}^{n N}\right)\right)$ are $h$-independent and in particular, $\pi_{00}$ is the spectral projector of $P^{c}(0)$ associated with a simple, stable eigenvalue $e_{0}$.

Theorem 5.3. Assume the conditions (3.1), (5.3) and the stability of $E_{0}(h)$. For any $b_{1}, b_{2} \in S$, c a bounded symbol over $\mathbf{R}^{2 n}$, put

$$
W(h)=b_{1}(x, h D) \Omega^{A D}(h)^{*} c(x, h D) \Omega^{A D}(h) b_{2}(x, h D) .
$$

Then $W(h)$ is an h-admissible operator (i.e., an h-pseudodifferential operator modulo an error of order $O\left(h^{\infty}\right)$, see [Ro]), and its leading symbol is given by

$$
w_{0}(x, \xi)=b_{1}(x, \xi) b_{2}(x, \xi) c\left(\Omega^{\mathrm{cl}}(x, \xi)\right) G(x, \xi) .
$$

Here $\Omega^{\mathrm{cl}}(x, \xi)$ is the outgoing classical wave operator for the classical Hamiltonians $\left(\xi^{2}, \xi^{2}+\Lambda_{0}(x)-e_{0}\right)$ and $G(x, \xi) \in \mathscr{C}\left(L^{2}\left(\mathbf{R}^{n N}\right)\right)$ is defined by

$$
G(x, \xi)=\pi_{00} G_{0}^{*}(y, \xi) \pi_{0}(y) G_{0}(y, \xi) \pi_{00}, \quad y=y(x+2 t \xi, \xi),
$$

for $t>T$, with $G_{0}$ given by (5.14) and $y(x, \xi)$ denoting the inverse of the global diffeomorphism $x \mapsto \nabla_{x} \phi(x, \xi)$.

Proof. We construct $J(a)$ such that (5.20) holds for $b_{1}$ and $b_{2}$. Then one has for $t>T$, $T$ sufficiently large

$$
W(h)=b_{1}(x, h D) W(t, h)^{*} c(x, h D) W(t, h) b_{2}(x, h D)+O\left(h^{\infty}\right) .
$$

By Egorov's theorem and the calculus of Fourier integral operators (see [W1]), one sees that $W(t, h)^{*} c(x, h D) W(t, h)$ is an $h$-pseudodifferential operator with operatorvalued symbol. To compute the leading symbol of $W(h)$, we proceed as in [W3, Proposition 4.5]. The difference from [W3] is that we now have to consider the operator-valued symbol $G_{0}(x, \xi)$ defined in (5.14). Note that for fixed $t>T$ the leading symbol of $J(a)^{*} U_{A D}(T) c(x, h D) U_{A D}(t)^{*} J(a)$ is

$$
d_{t}(x, \xi)=a_{0}(y, \xi)^{*} a_{0}(y, \xi)\left|\partial_{x} y(x, \xi)\right| c\left(\Phi^{-t}\left(y, \nabla_{x} \phi(y, \xi)\right)\right), \quad y=y(x, \xi),
$$

where $\phi(x, \xi)$ is a solution of the eikonal equation (5.9). For $(x, \xi) \in \operatorname{supp} b_{j}, j=1,2$, $\Phi_{0}^{-t}(x, \xi)$ is in the outgoing region $\Omega(\varepsilon, d, R)$. It then follows from the computation in [W3] that

$$
\begin{aligned}
d_{t}\left(\Phi_{0}^{t}(x, \xi)\right) & =H(x+2 t \xi, \xi) c\left(\Omega^{\mathrm{cl}}(x, \xi)\right), \\
H(x, \xi) & =\pi_{00} G_{0}(y, \xi)^{*} \pi_{0}(y) G_{0}(y, \xi) \pi_{00}, \quad y=y(x, \xi),
\end{aligned}
$$

for $(x, \xi) \in \operatorname{supp} b_{\jmath}, j=1,2$, and $t>T$.

In the derivation of (5.24), we used the following expression for the classical wave operator:

$$
\Omega^{\mathrm{cl}}(x, \xi)=\left(y(x, \xi),\left(\nabla_{x} \phi\right)(y(x, \xi), \xi), \quad(x, \xi) \in \Omega(2 \varepsilon, 2 d, 2 R) .\right.
$$

We refer to [W3] for a proof of this relation. Now Eq. (5.22) follows easily.

One can verify that the leading symbol $w_{0}$ of $W(h)$ is in fact independent of $t$ for $t>T$, if $T$ is sufficiently large (see the proof of Theorem 5.4 below, which gives $G(x, \xi)=\pi_{00}$ for $T$ large enough and for $\left.\xi \neq 0\right)$.

To obtain the classical limit of the adiabatic wave operators, we study its action on coherent states. Let $U_{h}$ denote the dilation

$$
U_{h} f(x)=h^{-n / 4} f\left(h^{-1 / 2} x\right), \quad f \in L^{2}\left(\mathbf{R}_{x}^{n}, L^{2}\left(\mathbf{R}^{n N}\right)\right) .
$$


For $\left(x_{0}, \xi_{0}\right) \in \mathbf{R}^{2 n}, \xi_{0} \neq 0$, put

$$
W_{h}\left(x_{0}, \xi_{0}\right)=U_{h} e^{2 h^{-1 / 2}\left(x \cdot \xi_{0}-x_{0} \cdot D_{x}\right)} .
$$

Take $\chi \in C_{0}^{\infty}\left(\mathbf{R}_{+}\right)$with $\chi\left(\xi_{0}^{2}\right)=1$.

Theorem 5.4. Assume (3.1) and (5.3). Let $\Omega^{A D}(h)$ be the adiabatic wave operator associated to the simple eigenvalue $\lambda_{1}(x, h)$ of $P_{e}(x, h)$ such that

$$
\lim _{|x| \rightarrow \infty} \lambda_{1}(x, h)=E_{0}(h),
$$

where $E_{0}(h)$ is a stable eigenvalue of $P^{c}(h)$. Then, for any bounded symbol $c$ and any $\left(x_{0}, \xi_{0}\right) \in \mathbf{R}^{2 n}, \xi_{0} \neq 0$, one has

$$
\lim _{h \rightarrow 0} F(h) f=c\left(\Omega^{\mathrm{cl}}\left(x_{0}, \xi_{0}\right)\right) \pi_{00} f, \quad f \in L^{2}\left(\mathbf{R}^{n(N+1)}\right),
$$

where

$$
F(h)=W_{h}\left(x_{0}, \xi_{0}\right)^{*} \chi\left(P_{0}(h)\right) \Omega^{A D}(h)^{*} c(x, h D) \Omega^{A D}(h) \chi\left(P_{0}(h)\right) W_{h}\left(x_{0}, \xi_{0}\right),
$$

and $\Omega^{\mathrm{cl}}$ is the outgoing classical wave operator for the classical Hamiltonians $\left(\xi^{2}, \xi^{2}+\Lambda_{0}(x)-e_{0}\right)$ and $P_{0}(h)=-h^{2} \Delta$.

Proof. It suffices to prove (5.27) for $f$ in the dense subset $C_{0}^{\infty}\left(\mathbf{R}^{n(N+1)}\right)$. Let $\chi_{1}$ be in $C_{0}^{\infty}\left(\mathbf{R}_{x}^{n}\right)$ with $\chi_{1}(x)=1$ for $|x|<1$. Put $\chi_{h}(x)=\chi_{1}\left(h^{1 / 2} x\right)$. For $f \in C_{0}^{\infty}\left(\mathbf{R}^{n(N+1)}\right)$ we can verify that

$$
\lim _{h \rightarrow 0}\left(\chi_{h} F(h) \chi_{h} f-F(h) f\right)=0 .
$$

Since

$$
W_{h}\left(x_{0}, \xi_{0}\right) \chi_{h} W_{h}\left(x_{0}, \xi_{0}\right)^{*}=\chi_{1}\left(x-x_{0}\right)
$$

and since $\chi_{1}\left(x-x_{0}\right) \chi\left(\xi^{2}\right)$ is in $S$, we can apply Theorem 5.3 to approximate $\chi_{h} F(h) \chi_{h}$ and conclude as in [W3] that

$$
\begin{aligned}
\lim _{h \rightarrow 0} F(h) f & =\chi_{1}(0) \chi\left(\xi_{0}^{2}\right) c\left(\Omega^{\mathrm{cl}}\left(x_{0}, \xi_{0}\right)\right) G\left(x_{0}, \xi_{0}\right) f \\
& =c\left(\Omega^{\mathrm{cl}}\left(x_{0}, \xi_{0}\right)\right) G\left(x_{0}, \xi_{0}\right) f .
\end{aligned}
$$

This proves the existence of the strong limit s- $\lim _{h \rightarrow 0} F(h)$. To show that $G\left(x_{0}, \xi_{0}\right)=\pi_{00}$, we note that $F(h)$ is $t$-independent and we can take the limit $t \rightarrow \infty$ in the definition (5.23) of $G\left(x_{0}, \xi_{0}\right)$. Combining (5.14) and the fact that $\pi_{0}(x) \rightarrow \pi_{00}$ as $x \rightarrow \infty$, we get that $\lim _{t \rightarrow \infty} G\left(x_{0}, \xi_{0}\right)=\pi_{00}$. This proves the theorem.

Note that the argument used in the proof of Theorem 5.4 shows also that $w_{0}$ in (5.22) is in fact $t$-independent for $t>T$.

We emphasize that the non-trapping condition on the classical effective Hamiltonian is not needed in Theorems 5.3 and 5.4. This should not be surprising, since the classical wave operator $\Omega^{\text {cl }}(x, \xi)$ is always well defined on $\mathbf{R}_{x}^{n} \times\left(\mathbf{R}_{\xi}^{n} \backslash\{0\}\right)$. Note that the main assumption in Theorems 5.3 and 5.4 is that $\Pi(x, h)$ is a globally defined spectral projector with rank one. In particular, these results apply to excited states $E_{0}(h)=e_{0}+O\left(h^{2}\right)$, where $e_{0}$ is a simple, stable eigenvalue of $P^{c}(0)$. Returning now to the cluster wave operators $\Omega_{ \pm}^{c}(h)$, we easily derive from Corollary 4.5 and Theorem 5.4 the following 
Theorem 5.5. Under the assumptions of Theorem 3.4 with $m=1$, let $\left(x_{0}, \xi_{0}\right) \in \mathbf{R}^{2 n}$ with $\xi_{0}^{2}=\lambda_{0}$, where $\lambda_{0}>0$ is a non-trapping energy of $p(x, \xi)=\xi^{2}+\Lambda_{0}(x)-e_{0}$. Then we have, for any bounded symbol $c$ on $\mathbf{R}_{x \xi}^{2 n}$ and any $\chi \in C_{0}^{\infty}(\mathbf{R})$ with support near $\lambda_{0}$ and $\chi\left(\lambda_{0}\right)=1$, that

$$
\begin{aligned}
& s-\lim _{h \rightarrow 0} W_{h}\left(x_{0}, \xi_{0}\right)^{*} \chi\left(P_{0}(h)\right) \Pi_{0}(h) \Omega_{ \pm}^{c}(h)^{*} c(x, h D) \Omega_{ \pm}^{c}(h) \Pi_{0}(h) \chi\left(P_{0}(h)\right) W_{h}\left(x_{0}, \xi_{0}\right) \\
& \quad=c\left(\Omega_{ \pm}^{\mathrm{cl}}\left(x_{0}, \xi_{0}\right)\right) \pi_{00},
\end{aligned}
$$

where $\Omega_{ \pm}^{\mathrm{cl}}$ are the classical outgoing wave operators associated to the effective Hamiltonian $p(x, \xi)$ and $\pi_{00}$ is the spectral projection of $P^{c}(0)$ associated to its first eigenvalue $e_{0}$.

To conclude this paper, we remark that we have only treated a restricted class of potentials (smooth short-range potentials) with a stability assumption on the eigenvalue of the cluster Hamiltonian $P^{c}$. Many problems related to BornOppenheimer approximation of molecular scattering theory remain open. We hope that this work could serve to initiate a mathematical study of Born-Oppenheimer approximation in molecular scattering, a subject which is of interest in molecular physics.

Acknowledgements. M.K. wants to thank R. Seiler for helpful discussions and J.M. Combes for explaining a long time ago at Berlin how the coordinates used in this paper and in [Ra] could be useful for discussing the Born-Oppenheimer approximation in scattering theory.

\section{References}

[A] Agmon, S.: Lectures on exponential decay of solutions of second-order elliptic equations. Princeton, NJ: Princeton University Press 1982

[BO] Born, M., Oppenheimer, R.: Zur Quantentheorie der Moleküln. Ann. Physik 84, 457 (1927)

[CDS] Combes, J.M., Duclos, P., Seiler, R.: The Born-Oppenheimer approximation. Rigorous Atomic and Molecular Physics. Wightman, A.S. and Velo, G., eds. New York: Plenum 1981

[Ch] Child, M.S.: Semiclassical methods in molecular scattering and spectroscopy. Proc. of NATO conferences, D. Reidel 1980

[De] Delos, J.B.: Theory of electronic transitions in slow atomic collisions. Rev. Mod. Phys. 53, 287-358 (1981)

[DH] Duclos, P., Hogreve, H.: On the stability of positive diatomic molecular ions. Preprint 1990

[GM] Gerard, C., Martinez, A.: Principe d'absorption limite pour des opérateurs de Schrödinger à longue portée. C. R. Acad. Sci. 306, 121-123 (1988)

[HS1] Helffer, B., Sjostrand, J.: Multiple wells in the semiclassical limit 1. Comm. PDE 9, 337-408 (1984)

[HS2] Helffer, B., Sjostrand, J.: Opérateurs de Schrödinger avec champs magnétiques faibles et constants. Exposé No. XII, Séminaire EDP, février 1989, Ecole Polytechniques

[IK] Isozaki, H., Kitada, H.: Modified wave operators with time-dependent modifiers. J. Fac. Sci. Univ. Tokyo, 32, 77-104 (1985)

[K] Kato, T.: Perturbation theory for linear operators. Berlin, Heidelberg, New York: Springer 1976

[KMSW] Klein, M., Martinez, A., Seiler, R., Wang, X.P.: On the Born-Oppenheimer expansion for polyatomic molecules. Commun. Math. Phys. 143, 607-639 (1992)

[M] Mourre, E.: Absence of singular continuous spectrum for certain self-adjoint operators. Commun. Math. Phys. 78, 391-408 (1981)

[Ra] Raphaelian, A.: Ion-atom scattering within a Born-Oppenheimer framework. Dissertation TU Berlin 1986 
[RS] Reed, M, Simon, B.: Methods of modern mathematical physics 3. New York: Academic Press 1979

[Ro] Robert, D.: Autour de l'approximation semiclassique. Progress in Math., No. 68. Basel, Boston: Birkhäuser 1987

[RT] Robert, D., Tamura, H.: Semiclassical estimates for resolvents and asymptotics for total scattering cross-section. Ann. IHP 46, 415-442 (1987)

[W1] Wang, X.P.: Time-decay of scattering solutions and resolvent estimates for semiclassical Schrödinger operators. J. Diff. Equations 71, 348-395 (1988)

[W2] Wang, X.P.: Time-decay of scattering solutions and classical trajectories. Ann. IHP 47, 25-37 (1987)

[W3] Wang, X.P.: Time-delay operators in semiclassical limit 2.Short-range potentials. Trans. AMS 322, 395-415 (1990)

[W4] Wang, X.P.: Semiclassical resolvent estimates for N-body Schrödinger operators. J. Funct. Anal. 97, 466-483 (1991)

[WO] Wu, T.Y., Ohmura, T.: Quantum theory of scattering. Prentice-Hall 1962

$[Y] \quad Y a j i m a, K .:$ The quasi-classical limit of quantum scattering theory. Commun. Math. Phys. 69, 101-129 (1979)

Communicated by B. Simon 
